\title{
In Vitro Antimicrobial Activity and Effect on Biofilm Production of a White Grape Juice (Vitis vinifera) Extract
}

\author{
Angela Filocamo, Carlo Bisignano, Giuseppina Mandalari, and Michele Navarra \\ Department of Chemical, Biological, Pharmaceutical and Environmental Science, University of Messina, \\ Viale Annunziata, 98168 Messina, Italy \\ Correspondence should be addressed to Giuseppina Mandalari; gmandalari@unime.it and Michele Navarra; mnavarra@unime.it
}

Received 18 August 2015; Revised 10 November 2015; Accepted 23 November 2015

Academic Editor: Michał Tomczyk

Copyright (C) 2015 Angela Filocamo et al. This is an open access article distributed under the Creative Commons Attribution License, which permits unrestricted use, distribution, and reproduction in any medium, provided the original work is properly cited.

Background. The aim of the present study was to evaluate the antimicrobial effect of a white grape juice extract (WGJe) against a range of Gram-positive and Gram-negative bacteria, yeasts, and the fungus Aspergillus niger. WGJe was also tested on the production of bacterial biofilms in vitro. Results. WGJe inhibited in vitro most Gram-positive bacteria tested, Staphylococcus aureus ATCC 6538P being the most sensitive strain (MIC values of $3.9 \mu \mathrm{g} / \mathrm{mL}$ ). The effect was bactericidal at the concentration of $500 \mu \mathrm{g} / \mathrm{mL}$. Amongst the Gram-negative bacteria, Escherichia coli was the only susceptible strain (MIC and MBC of $2000 \mu \mathrm{g} / \mathrm{mL}$ ). No effect on the growth of Candida sp. and the fungus Aspergillus niger was detected (MIC values $>2000 \mu \mathrm{g} / \mathrm{mL}$ ). WGJe inhibited the biofilms formation of E. coli and Pseudomonas aeruginosa with a dose-dependent effect. Conclusions. WGJe exerted both bacteriostatic and bactericidal activity in vitro. The presented results could be used to develop novel strategies for the treatment of skin infections and against potential respiratory pathogens.

\section{Introduction}

A wide variety of compounds have been identified in grapes, most of which with health promoting properties. A number of studies have shown that beneficial effects of grape and grape products consumption are related to the presence of polyphenols, mainly flavonoids and phenolic acids, with antioxidant, anti-inflammatory, antimicrobial, antiviral, and cancer preventive properties [1-4]. Flavonoids, stilbene, and proanthocyanidins are considered the most abundant class of bioactive compounds in grapes. It has also been demonstrated that grape juices, both white and purple, represent an important source of minerals, which would help explain the antioxidant and antimutagenic properties of grapes [5]. We have recently shown that an extract from white grape juice could have a beneficial effect on radiocontrast medium toxicity in human renal proximal tubular cells [6] and exert neuroprotective effect in a mouse model of experimental autoimmune encephalomyelitis [7]. Due to the increased antibiotic resistance, the antimicrobial properties of natural compounds have been gaining attention: we have previously reported the antibacterial activity of polyphenols-rich natural products, including almonds [8, 9], pistachios [10], Citrus plants [11], Vitis vinifera L. [12], Olea europaea L. [13], Citrus bergamia essential oil [14], and juice [15]. In particular, the identification of novel compounds with bactericidal rather than bacteriostatic effect has attracted interest in recent years. Inhibition and eradication of Gram-positive and Gram-negative biofilms have also been proven to be rather difficult with conventional antibiotics, with a need for novel antimicrobials able to treat biofilm-related infections. Yadav et al. [16] have recently demonstrated that eugenol was effective against methicillin-resistant and methicillinsensitive Staphylococcus aureus clinical strain biofilms. The effects of cranberry extracts have also been evaluated on the growth and biofilm production of Escherichia coli and Staphylococcus sp. [17].

In the present study we evaluated the antimicrobial effect of a polyphenols-rich white grape juice extract against Grampositive and Gram-negative bacteria, yeasts, and the fungus Aspergillus niger. Furthermore, the same extract was tested on the production of bacterial biofilms in vitro. 


\section{Materials and Methods}

2.1. Grape Juice Extract. A white grape (Vitis vinifera) juice extract (WGJe) was kindly provided by "Bono \& Ditta" (Campobello di Mazzara, Trapani, Italy). The extract derived from a mixture of white grapes juice from Vitis vinifera var. Catarratto, Vitis vinifera var. Grillo, and Vitis vinifera var. Insolia. WGJe was produced in its liquid form by passing the must-mute columns equipped with adsorbent resins, known to retain polyphenols. Molecules were then eluted with $4 \%$ $\mathrm{NaOH}$ and immediately passed through cationic resins, thus obtaining an acidic form. Products were collected, filtered, and then sprayed to obtain a dry powder kept at $-20^{\circ} \mathrm{C}$ until further use $[6,7]$. WGJe chemical composition, evaluated by UPLC/QqQ-MS/MS, has been previously reported [6, 7]. The major polyphenols identified in WGJe were quercetin3-glucuronide, procyanidin B1, quercetin-3-glucoside, catechin, and $t$-coutaric acid. Minor identified compounds included a number of glucosides, such as kaempferol-3glucuronide and kaempferol-3-glucoside, isorhamnetin-3glucoside, and quercetin-3-glucoside-arabinoside. Phenolic acids, including vanillic acid, ellagic acid, ferulic acid, chlorogenic acid, caffeic acid, and $p$-coumaric acid, were also identified.

2.2. Microbial Strains and Culture Conditions. The following strains from the University of Messina's in-house culture collection (Messina, Italy) were used for the antimicrobial testing: Staphylococcus aureus ATCC 6538P, Staphylococcus aureus MRSA ATCC 43300, Staphylococcus epidermidis ATCC 49134, Staphylococcus epidermidis ATCC 35984, Staphylococcus epidermidis ATCC 12228, Streptococcus pneumoniae ATCC 6003, Streptococcus pyogenes ATCC 19615, Streptococcus mutans ATCC 35668, Listeria monocytogenes ATCC 7644, Listeria monocytogenes ATCC 1392, Enterococcus hirae ATCC 10541, Moraxella catarrhalis ATCC 8176, Bacillus subtilis ATCC 8176, Enterococcus durans (wildtype strain), Escherichia coli ATCC 25922, Klebsiella pneumoniae (wild-type strain), Pseudomonas aeruginosa ATCC 27853, Pseudomonas aeruginosa ATCC 9027, Pseudomonas aeruginosa ATCC 15442, Proteus mirabilis (wild-type strain), Serratia marcescens (wild-type strain), Salmonella typhi ATCC 13311, Candida albicans ATCC 10231, Candida parapsilosis ATCC 29947, and the fungus Aspergillus niger ATCC 16404.

Bacteria were grown in Mueller-Hinton Broth (MHB, Oxoid, CM0405) at $37^{\circ} \mathrm{C}(24 \mathrm{~h})$, whereas yeasts were cultured in Sabouraud Liquid Medium (SLM, Oxoid, CM0147) at $30^{\circ} \mathrm{C}$ $(48 \mathrm{~h})$. For solid media, $1.5 \%$ (w/v) agar (Difco) was added. Aspergillus niger was grown in Sabouraud Dextrose Agar at $30^{\circ} \mathrm{C}$ for 7 days as previously reported [10].

2.3. Susceptibility Studies. For the susceptibility studies, WGJe was dissolved in sterile PBS at the concentration of $4 \mathrm{mg} / \mathrm{mL}$; the $\mathrm{pH}$ of this solution was 7.10 . The minimum inhibitory concentration (MIC), the minimum bactericidal concentration (MBC), and the minimum fungicidal concentration (MFC) of WGJe were determined by the broth microdilution method, according to CLSI [18]. Briefly, twofold serial dilutions of WGJe were added to MHB and inoculated into 96-microtiter plates with a final inoculum of approximately $5 \times 10^{5} \mathrm{CFU} \mathrm{mL}^{-1}$. The tested concentrations ranged from 2000 to $3.9 \mu \mathrm{g} \mathrm{mL}^{-1}$, and no significant changes of the $\mathrm{pH}$ of the growth medium were detected after addition of WGJe. The MIC was considered as the lowest concentration, which completely inhibited bacterial growth after $20 \mathrm{~h}$.

The MBCs were determined by seeding $20 \mu \mathrm{L}$ from all clear MIC wells onto Mueller-Hinton agar (MHA, Oxoid) plates. The MBC was defined as the lowest extract concentration that killed $99.9 \%$ of the final inocula after $24 \mathrm{~h}$ incubation.

2.4. Effect on Biofilm Formation. The effect of different concentrations of WGJe on biofilm-forming ability was tested on polystyrene flat-bottomed microtiter plates as described by Nostro et al. [19].

Twofold serial dilutions of WGJe ranging from 500 to $62.5 \mu \mathrm{g} \mathrm{mL}^{-1}$ were used for E. coli ATCC 25922 and $P$. aeruginosa ATCC 9027.

Cultures were grown overnight and suspensions adjusted to $10^{6} \mathrm{CFUmL}^{-1}$. Aliquots of $100 \mathrm{~mL}$ were dispensed into each well of a sterile flat-bottomed 96-well polystyrene microtiter plates (Corning Inc., Corning, NY) in the presence of WGJe.

Planktonic growth was determined by spectrophotometric values $\left(\mathrm{OD}_{492} \mathrm{~nm}\right)$ after $24 \mathrm{~h}$. The medium was then aspirated and the wells, rinsed twice with phosphate-buffered saline (PBS), were fixed by drying for $2 \mathrm{~h}$. Once the wells were fully dry, $200 \mathrm{~mL}$ of $0.1 \%$ safranin was added for $2 \mathrm{~min}$. The content of the wells was then aspirated, and, after rinsing with water, $200 \mathrm{~mL}$ of $30 \%$ acetic acid (v/v) was added. $\mathrm{OD}_{492}$ was measured by spectrophotometry using an ELISA reader.

Biofilm controls consisting of medium alone, medium plus strains (without WGJe), and medium plus WGJe (without strain) were included.

Each assay was performed in duplicate and repeated at least three times.

\section{Results}

3.1. Minimum Inhibitory Concentrations. The MIC and MBC values of WGJe against the strains tested are shown in Table 1. WGJe was active against all the Gram-positive strains included in the study (MIC values between 3.9 and $1000 \mu \mathrm{g} \mathrm{mL}^{-1}$ ), whereas no activity was found against the Gram-negative bacteria, with the exception of E. coli (MIC of $2000 \mu \mathrm{g} \mathrm{mL}^{-1}$ ), the yeasts, and A. niger at the concentrations tested.

Amongst the Gram-positive bacteria, S. aureus ATCC $6538 \mathrm{P}$ showed the highest sensitivity, with MIC and MBC values equal to 3.9 and $500 \mu \mathrm{g} \mathrm{mL}^{-1}$, respectively.

Similarly, a good growth inhibitory activity was found against $S$. epidermidis and $M$. catarrhalis, with MIC values of $15.62 \mu \mathrm{g} \mathrm{mL}^{-1}$ and MBC values of $2000 \mu \mathrm{g} \mathrm{mL}^{-1}$. WGJe $\left(125 \mu \mathrm{g} \mathrm{mL}^{-1}\right)$ has also been able to inhibit the growth of $L$. monocytogenes, S. mutans, S. pyogenes, and S. pneumonia. $\mathrm{MBC}$ values of $1000 \mu \mathrm{g} \mathrm{mL}^{-1}$ for $S$. pyogenes and S. pneumonia and $2000 \mu \mathrm{g} \mathrm{mL}^{-1}$ for S. mutans were detected, whereas 
TABLE 1: MICs and MBCs of WGJe (expressed as $\mu \mathrm{g} / \mathrm{mL}$ ) against Gram-positive bacteria, Gram-negative bacteria, yeasts, and the fungus $A$. niger.

\begin{tabular}{|c|c|c|}
\hline & MIC & MBC \\
\hline \multicolumn{3}{|l|}{ Gram+ } \\
\hline M. catarrhalis ATCC 8176 & 15.62 & 2000 \\
\hline B. subtilis ATCC 6633 & 250 & 2000 \\
\hline E. durans (wild-type strain) & 500 & $>2000$ \\
\hline E. hirae ATCC 10541 & 1000 & $>2000$ \\
\hline L. monocytogenes ATCC 7466 & 125 & $>2000$ \\
\hline L. monocytogenes ATCC 13932 & 250 & $>2000$ \\
\hline S. aureus ATCC 6538P & 3.9 & 500 \\
\hline S. aureus ATCCC 43300 & 62.5 & 500 \\
\hline S. epidermidis ATCC 49134 & 15.62 & $>2000$ \\
\hline S. epidermidis ATCC 12228 & 31.25 & 2000 \\
\hline S. epidermidis ATCC 35984 & 15.62 & 2000 \\
\hline S. mutans ATCC 35668 & 125 & 2000 \\
\hline S. pyogenes ATCC 19615 & 125 & 1000 \\
\hline S. pneumoniae ATCC 6003 & 125 & 1000 \\
\hline \multicolumn{3}{|l|}{ Gram- } \\
\hline E. coli ATCC 25922 & 2000 & 2000 \\
\hline K. pneumoniae (wild-type strain) & $>2000$ & $>2000$ \\
\hline P. aeruginosa ATCC 27853 & $>2000$ & $>2000$ \\
\hline P. aeruginosa ATCC 9027 & $>2000$ & $>2000$ \\
\hline P. aeruginosa ATCC15442 & $>2000$ & $>2000$ \\
\hline P. mirabilis (wild-type strain) & $>2000$ & $>2000$ \\
\hline S. marcescens (wild-type strain) & $>2000$ & $>2000$ \\
\hline S. typhi ATCC13311 & $>2000$ & $>2000$ \\
\hline \multicolumn{3}{|l|}{ Yeasts } \\
\hline C. albicans ATCC 10231 & $>2000$ & $>2000$ \\
\hline C. parapsilosis ATCC 29947 & $>2000$ & $>2000$ \\
\hline \multicolumn{3}{|l|}{ Fungi } \\
\hline A. niger ATCC 16404 & $>2000$ & $>2000$ \\
\hline
\end{tabular}

MICs, minimal inhibitory concentrations; MBCs, minimal bactericidal concentrations; WGJe, white grape juice extract.

no bactericidal activity was found against $L$. monocytogenes at the concentrations tested.

MIC values of 250,500 , and $1000 \mu \mathrm{g} \mathrm{mL}^{-1}$ were also found against $B$. subtilis, E. durans, and E. hirae, respectively. A MBC value corresponding to the maximum concentration tested was detected for B. subtilis, whereas the same concentration showed bacteriostatic activity on E. durans and E. hirae.

Amongst the Gram-negative bacteria, a slight inhibitory activity was found against $E$. coli for which the value of MIC found, however, corresponds to the highest concentration used. No activity was observed against other Gram-negative strains.

3.2. Effect on Biofilm Formation. Table 2 shows the effect of WGJe on the biofilm formation of E. coli and P. aeruginosa. Since the MIC value was relatively high for E. coli and no inhibition was observed against $P$. aeruginosa (Table 1), the WGJe concentrations used ranged between 500 and $62.5 \mu \mathrm{g} \mathrm{mL}^{-1}$.

The results demonstrated that WGJe (500 and $250 \mu \mathrm{g} \mathrm{mL}^{-1}$ ) produced a reduction on biofilm formation of $42.49 \%$ and $23.79 \%$ for $E$. coli and $30.47 \%$ and $23.88 \%$ for $P$. aeruginosa, respectively.

\section{Discussion}

The present study has demonstrated that WGJe was effective against a range of Gram-positive bacteria. Although WGJe did not affect the growth of any Gram-negative bacteria tested, with the exception of E. coli, a reduction on biofilm formation of both E. coli and P. aeruginosa was detected. A number of studies have previously investigated the antimicrobial effect of grape, wine, and their byproducts [20, 21]. Red wine has been shown to prevent damage to the gastric mucosa induced by Helicobacter pylori, possibly through inhibition of the vacA gene [22]. Jayaprakasha et al. [23] have demonstrated that grape seed extracts have antimicrobial potential, Gram-positive bacteria being more sensitive than Gram-negative bacteria. Our study has also demonstrated that Gram-positive strains were more susceptible to WGJe compared with Gram-negative bacteria, with a bactericidal effect observed on all Gram-positive strains tested, with the exception of E. durans, E. hirae, Listeria spp., and S. epidermidis ATCC 49134. A strong inhibitory effect against Listeria monocytogenes has been found by grape juice and grape extracts derived from Vitis vinifera variety "Ribier" [24]. Sanhueza et al. [25] have recently reported an antibacterial effect of grape pomace extracts mainly against $S$. aureus and E. coli: the activity was directly related to the polar phenolic content. Grape seed extracts obtained from wine and table cultivars of Vitis vinifera L. were found to be active against Candida albicans sp. and their activity was related to the presence of polymeric flavan-3-ols [26].

The antibacterial activity of polyphenols has been recently reviewed [27]. In agreement with our study, several wine phenolic acids, mainly gallic acid and ethyl gallate, were able to inhibit the growth of respiratory pathogenic bacteria and potential respiratory pathogens including $P$. aeruginosa, $S$. aureus, M. catarrhalis, and E. faecalis [28]. However, unlike the report by Cueva et al. [28], we found Gram-positive bacteria were more sensitive to WGJe compared with the Gram-negative tested strains. Some phenolic acids, such as cinnamic acid, ferulic acid, $p$-coumaric acid, and caffeic acid, were also found to be active against Listeria spp. [29]. Chlorogenic acid extracted from blueberry fruit was able to inhibit $46 \%$ and $42 \%$ of $S$. epidermidis and P. aeruginosa biofilm formation, respectively [30]. We believe that the phenolic acids present in WGJe played an important role in reducing biofilm formation of E. coli and P. aeruginosa, in a dose-dependent way.

\section{Conclusions}

The results of the present study demonstrated that WGJe was effective against a range of Gram-positive bacteria, including 
TABLE 2: Percentage biofilm reduction for twofold serial dilutions of WGJe ranging from 500 to $62.5 \mu \mathrm{g} \mathrm{mL} \mathrm{on}^{-1}$. coli ATCC 25922 and $P$. aeruginosa ATCC 9027.

\begin{tabular}{lcccc}
\hline & 500 & 250 & 125 & 62.5 \\
\hline E. coli ATCC 10536 & $42.49 \pm 0.75$ & $23.79 \pm 0.48$ & $19.28 \pm 0.33$ & $10.55 \pm 0.78$ \\
$P$. aeruginosa ATCC 9027 & $30.47 \pm 0.27$ & $23.88 \pm 0.14$ & $21.20 \pm 0.19$ & $17.38 \pm 0.37$ \\
\hline
\end{tabular}

WGJe, white grape juice extract.

potential respiratory pathogens, and E. coli amongst Gramnegative strains. The exerted activity was both bacteriostatic and bactericidal. Furthermore, WGJe was able to inhibit the biofilm formation of E. coli and P. aeruginosa in vitro.

\section{Conflict of Interests}

The authors declare that they have no conflict of interests.

\section{Authors' Contribution}

All authors read and approved the final paper.

\section{Acknowledgment}

Research was supported by grants from Sicily Region (PO FESR Sicilia 2007/2013, project “MEPRA," N. 133 of Linea d'Intervento 4.1.1.1, CUP G73F11000050004) to Michele Navarra.

\section{References}

[1] V. Georgiev, A. Ananga, and V. Tsolova, "Recent advances and uses of grape flavonoids as nutraceuticals," Nutrients, vol. 6, no. 1, pp. 391-415, 2014.

[2] J. K. Lin and M. S. Weng, "Flavonoids as nutraceuticals," in The Science of Flavonoids, E. Grotewold, Ed., pp. 213-238, Springer, Berlin, Germany, 2006.

[3] L. Le Marchand, "Cancer preventive effects of flavonoids-a review," Biomedicine and Pharmacotherapy, vol. 56, no. 6, pp. 296-301, 2002.

[4] C. D. Wu, "Grape products and oral health," Journal of Nutrition, vol. 139, no. 9, pp. 1818S-1823S, 2009.

[5] C. Dani, L. S. Oliboni, D. Pra et al., "Mineral content is related to antioxidant and antimutagenic properties of grape juice," Genetics and Molecular Research, vol. 11, no. 3, pp. 3154-3163, 2012.

[6] M. Andreucci, T. Faga, A. Pisani et al., "Reversal of radiocontrast medium toxicity in human renal proximal tubular cells by white grape juice extract," Chemico-Biological Interactions, vol. 229, pp. 17-25, 2015.

[7] S. Giacoppo, M. Galuppo, G. E. Lombardo et al., "Neuroprotective effects of a polyphenolic white grape juice extract in a mouse model of experimental autoimmune encephalomyelitis," Fitoterapia, vol. 103, pp. 171-186, 2015.

[8] G. Mandalari, C. Bisignano, M. D’Arrigo et al., "Antimicrobial potential of polyphenols extracted from almond skins," Letters in Applied Microbiology, vol. 51, no. 1, pp. 83-89, 2010.

[9] C. Bisignano, A. Filocamo, E. La Camera, S. Zummo, M. T. Fera, and G. Mandalari, "Antibacterial activities of almond skins on cagA-positive and-negative clinical isolates of Helicobacter pylori," BMC Microbiology, vol. 13, article 103, 2013.

[10] C. Bisignano, A. Filocamo, R. M. Faulks, and G. Mandalari, "In vitro antimicrobial activity of pistachio (Pistacia vera L.) polyphenols," FEMS Microbiology Letters, vol. 341, no. 1, pp. 6267, 2013.

[11] G. Mandalari, R. N. Bennett, G. Bisignano et al., "Antimicrobial activity of flavonoids extracted from bergamot (Citrus bergamia Risso) peel, a byproduct of the essential oil industry," Journal of Applied Microbiology, vol. 103, no. 6, pp. 2056-2064, 2007.

[12] C. Bisignano, A. Filocamo, G. Mandalari, and M. Navarra, "Effect of a white grape (Vitisvinifera L.) juice extract on the growth and biofilm production of methicillin-resistant and sensitive Staphylococcus species," Clinical Microbiology \& Case Reports, vol. 1, no. 2, article 016, 2015.

[13] C. Bisignano, A. Filocamo, G. Ginestra et al., "3,4-DHPEA-EA from Olea Europaea L. is effective against standard and clinical isolates of Staphylococcus sp." Annals of Clinical Microbiology and Antimicrobials, vol. 13, no. 1, article 24, 2014.

[14] P. M. Furneri, L. Mondello, G. Mandalari et al., "In vitro antimycoplasmal activity of citrus bergamia essential oil and its major components," European Journal of Medicinal Chemistry, vol. 52, pp. 66-69, 2012.

[15] A. Filocamo, C. Bisignano, N. Ferlazzo, S. Cirmi, G. Mandalari, and M. Navarra, "In vitro effect of bergamot (Citrus bergamia) juice against cagA-positive and-negative clinical isolates of Helicobacter pylori," BMC Complementary and Alternative Medicine, vol. 15, article 256, 2015.

[16] M. K. Yadav, S.-W. Chae, G. J. Im, J.-W. Chung, and J.-J. Song, "Eugenol: a phyto-compound effective against methicillinresistant and methicillin-sensitive Staphylococcus aureus clinical strain biofilms," PLoS ONE, vol. 10, no. 3, Article ID e0119564, 2015.

[17] K. L. LaPlante, S. A. Sarkisian, S. Woodmansee, D. C. Rowley, and N. P. Seeram, "Effects of cranberry extracts on growth and biofilm production of Escherichia coli and Staphylococcus species," Phytotherapy Research, vol. 26, no. 9, pp. 1371-1374, 2012.

[18] CLSI, "Clinical and Laboratory Standards Institute performance standards for antimicrobial susceptibility testing; twentieth informational supplement," Tech. Rep. M100-S22, Clinical and Laboratory Standards Institute (CLSI), Wayne, Pa, USA, 2012.

[19] A. Nostro, A. S. Roccaro, G. Bisignano et al., "Effects of oregano, carvacrol and thymol on Staphylococcus aureus and Staphylococcus epidermidis biofilms," Journal of Medical Microbiology, vol. 56, no. 4, pp. 519-523, 2007.

[20] A. V. S. Perumalla and N. S. Hettiarachchy, "Green tea and grape seed extracts-potential applications in food safety and quality," Food Research International, vol. 44, no. 4, pp. 827-839, 2011.

[21] E.-Q. Xia, G.-F. Deng, Y.-J. Guo, and H.-B. Li, "Biological activities of polyphenols from grapes," International Journal of Molecular Sciences, vol. 11, no. 2, pp. 622-646, 2010. 
[22] P. Ruggiero, G. Rossi, F. Tombola et al., "Red wine and green tea reduce $H$ pylori- or VacA-induced gastritis in a mouse model," World Journal of Gastroenterology, vol. 13, no. 3, pp. 349-354, 2007.

[23] G. K. Jayaprakasha, T. Selvi, and K. K. Sakariah, "Antibacterial and antioxidant activities of grape (Vitis vinifera) seed extracts," Food Research International, vol. 36, no. 2, pp. 117-122, 2003.

[24] P. L. Rhodes, J. W. Mitchell, M. W. Wilson, and L. D. Melton, "Antilisterial activity of grape juice and grape extracts derived from Vitis vinifera variety Ribier," International Journal of Food Microbiology, vol. 107, no. 3, pp. 281-286, 2006.

[25] L. Sanhueza, M. Tello, M. Vivanco, L. Mendoza, and M. Wilkens, "Relation between antibacterial activity against food transmitted pathogens and total phenolic compounds in grape pomace extracts from Cabernet Sauvignon and Syrah varieties," Advances in Microbiology, vol. 4, no. 5, pp. 225-232, 2014.

[26] G. Simonetti, A. R. Santamaria, F. D. D’Auria et al., "Evaluation of anti-Candida activity of Vitis vinifera L. seed extracts obtained from wine and table cultivars," BioMed Research International, vol. 2014, Article ID 127021, 11 pages, 2014.

[27] E. Coppo and A. Marchese, "Antibacterial activity of polyphenols," Current Pharmaceutical Biotechnology, vol. 15, no. 4, pp. 380-390, 2014.

[28] C. Cueva, S. Mingo, I. Muñoz-González et al., "Antibacterial activity of wine phenolic compounds and oenological extracts against potential respiratory pathogens," Letters in Applied Microbiology, vol. 54, no. 6, pp. 557-563, 2012.

[29] A. Wen, P. Delaquis, K. Stanich, and P. Toivonen, "Antilisterial activity of selected phenolic acids," Food Microbiology, vol. 20, no. 3, pp. 305-311, 2003.

[30] K. R. Zimmer, C. H. Blum-Silva, A. L. K. Souza et al., "The antibiofilm effect of blueberry fruit cultivars against Staphylococcus epidermidis and Pseudomonas aeruginosa," Journal of Medicinal Food, vol. 17, no. 3, pp. 324-331, 2014. 


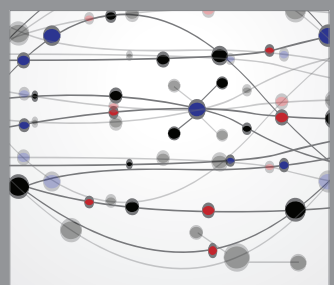

The Scientific World Journal
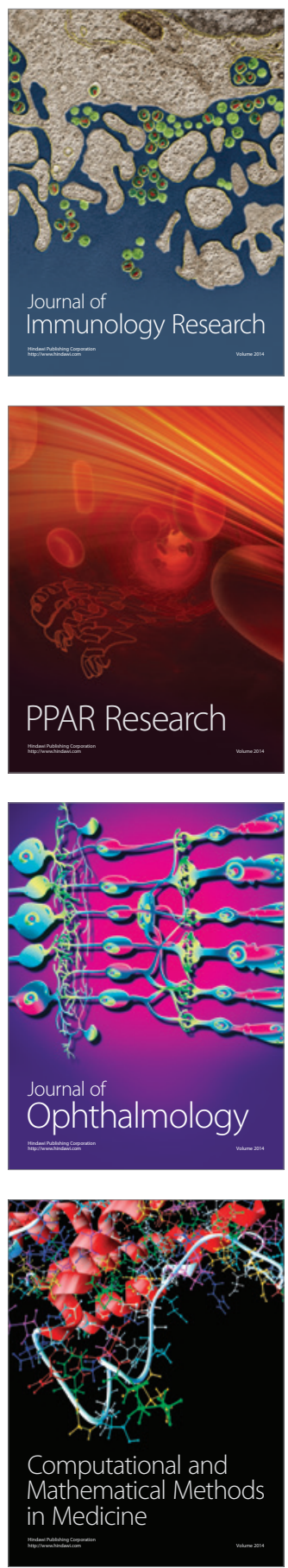

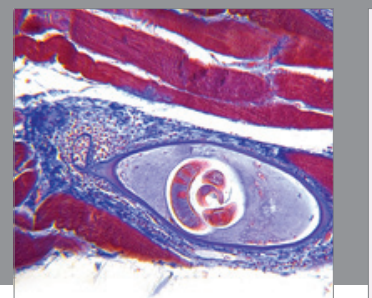

Gastroenterology

Research and Practice
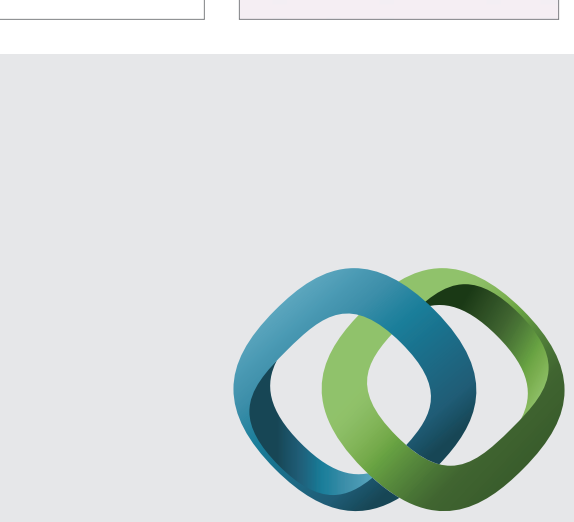

\section{Hindawi}

Submit your manuscripts at

http://www.hindawi.com
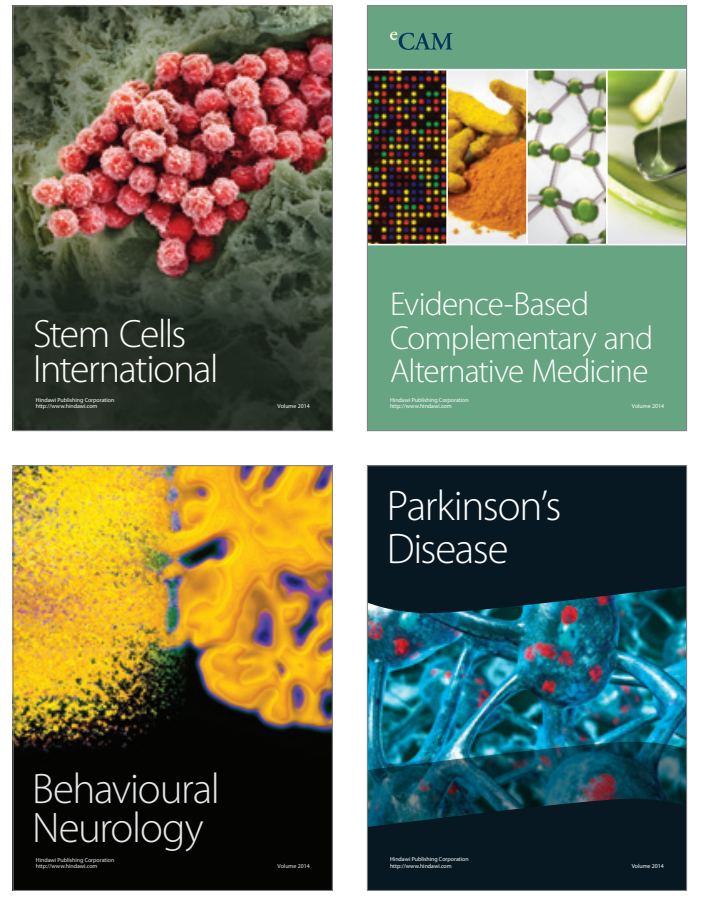
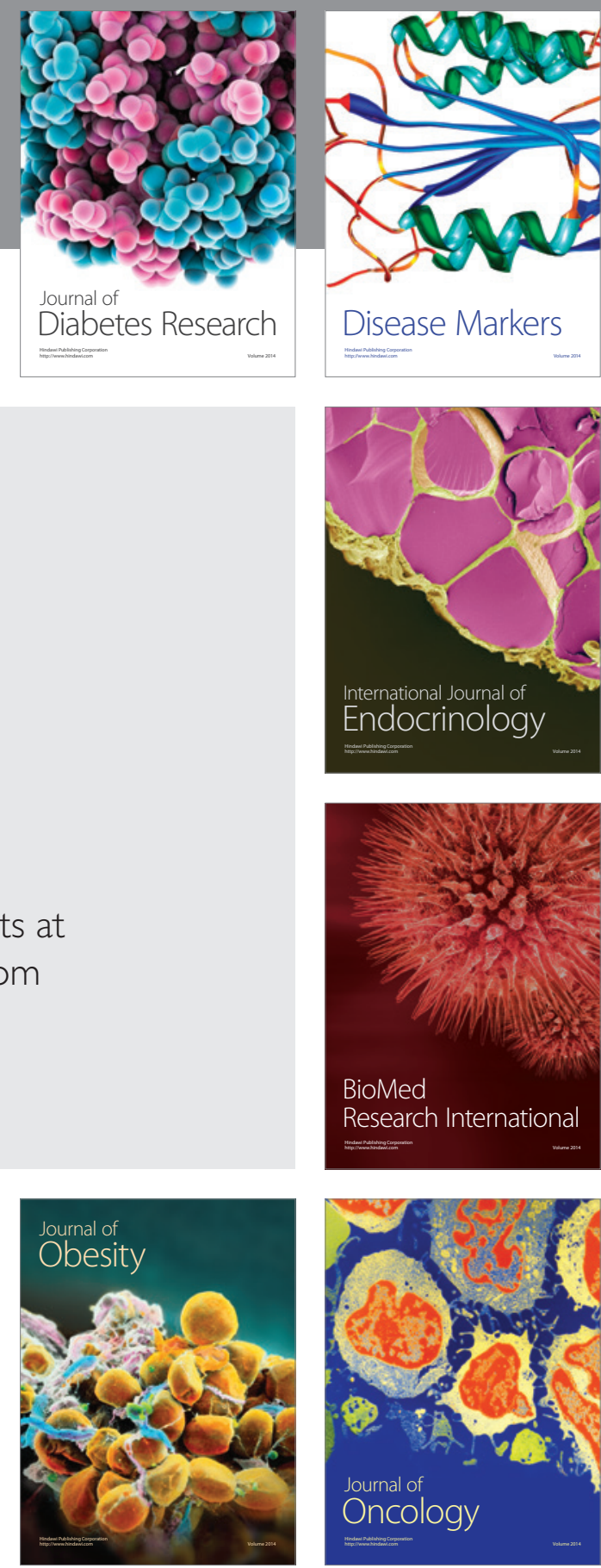

Disease Markers
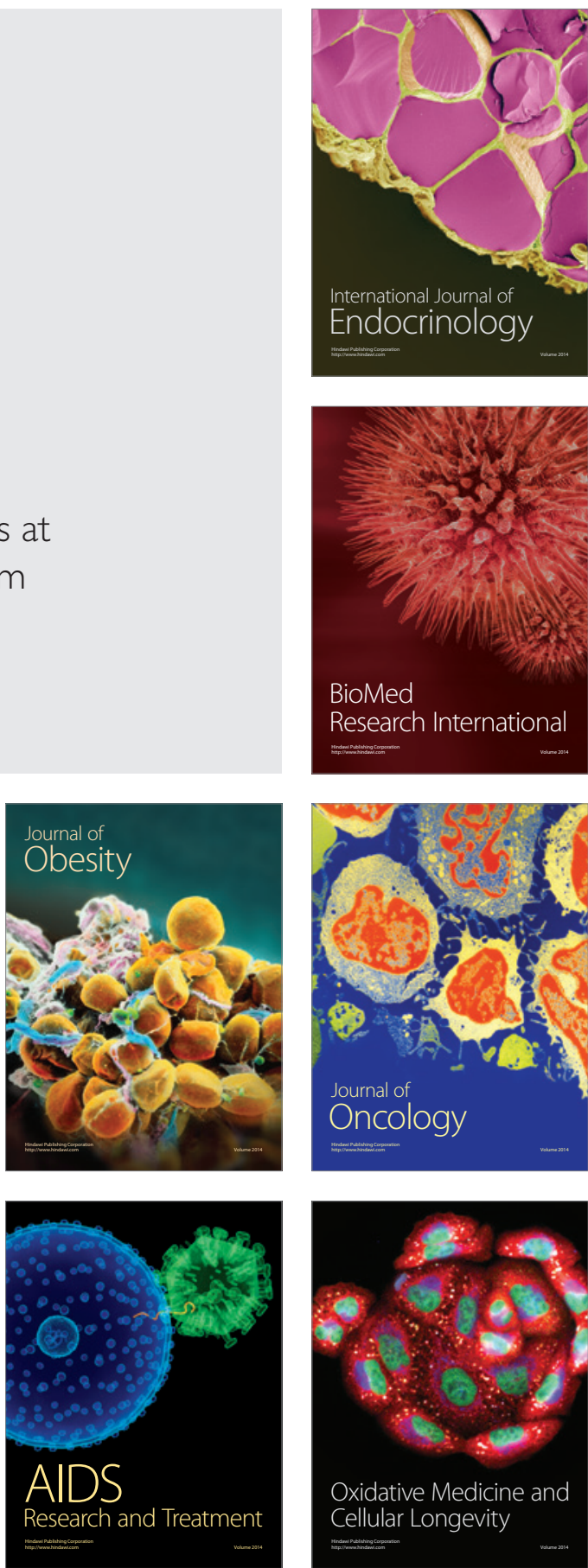